\title{
Temporal Encoding for Auditory Computation: Physiology of Primary Afferent Neurons in Sound-Producing Fish
}

\author{
Aae Suzuki, James Kozloski, and John D. Crawford \\ Department of Psychology and Neuroscience Graduate Group, University of Pennsylvania, Philadelphia, \\ Pennsylvania 19104
}

\begin{abstract}
Many fish rely on sounds for communication, yet the peripheral structures containing the hair cells are simple, without the morphological specializations that facilitate frequency analysis in the mammalian cochlea. Despite this, neurons in the midbrain of sound-producing fish (Pollimyrus) have complex receptive fields, extracting features from courtship sounds. Here we present an analysis of the initial encoding of sounds by the primary afferents and demonstrate that the representation of sound undergoes a substantial transformation as it ascends to the midbrain. Afferents were isolated as they coursed from the sacculus through the medulla. Tones $(100 \mathrm{~Hz}-1.2 \mathrm{kHz})$ elicited synchronized spikes [vector strength (VS) $>0.9$ ] on each stimulus cycle [coefficient of variation $(\mathrm{CV})<1.1$ ], with little spike rate adaptation. Most afferents (67\%) were spontaneously active and began synchronizing $10 \mathrm{~dB}$ below rate threshold. Rate thresholds for the most sensitive afferents (65 dB) were close to
\end{abstract}

behavioral thresholds. The distribution of characteristic frequencies and best sensitivities was matched to the spectrum of sounds of this species and to its audiogram. Three clusters of afferents were identified, one including afferents that generated spike bursts and had v-shaped response areas (bursters), and two others that included entrained afferents with broad response areas (entrained types I and II). All afferents encoded the timing of clicks within click trains with time-locked spikes, and none showed selectivity for interclick intervals. Understanding the computations that yield complex receptive fields is an essential goal for auditory neuroscience, and these data on primary encoding advance this goal, allowing a comparison of inputs with feature-extracting midbrain neurons.

Keywords: auditory communication; primary afferent; computation; electric fish; hearing; temporal processing; neural transformation; Mormyridae
In mammals, sound is place coded at the cochlea and carried into the brain through labeled lines. Within these channels, information about the temporal structure of sounds is encoded by synchronized spikes. Temporal analysis is particularly important at low frequencies $(<3 \mathrm{kHz})$, where spike synchronization is strongest and where speech and many other animal communication sounds are produced. Because temporal analysis predominates in fish, they serve as valuable model systems for time-domain processing in vertebrate hearing (Fay, 1978, 1982; Fay and Passow, 1982; Bodnar and Bass, 1997, 2001; McKibben and Bass, 1999).

The mormyrid fish Pollimyrus produces low-frequency communication sounds (Crawford et al., 1986, 1997a,b; Bratton and Kramer, 1989). The courtship displays of males are composed of alternating grunts [250 msec click trains, interclick interval (ICI) of $18 \mathrm{msec}$ ] and moans (800 msec tones, $250 \mathrm{~Hz}$ fundamental) (Crawford et al., 1997a). The sounds of closely related species are distinct, and the sounds of males, within a species, are also individually specific. Behaviorally, the fish are sensitive to small differences in click trains and tones, indicating that even minute

Received Aug. 29, 2001; revised April 29, 2002; accepted May 1, 2002.

This research was supported by National Institutes of Health Grant R01 DC01252 (J.D.C.), National Institute of Mental Health (NIMH) Grant PBN F31 MH11270 (J.K.), and NIMH Grant 5 F31 MH12510-02 (A.S.). A. P. Cook, L. A. Palmer, V. Richards, J. Saunders, D. Sparks, and P. Sterling provided valuable input during the research, and P. Marvit assisted with programming.

Correspondence should be addressed to Aae Suzuki, Department of Psychology and Neuroscience Graduate Group, University of Pennsylvania, 3815 Walnut Street, Philadelphia, PA 19104. E-mail: suzuki@mail.med.upenn.edu.

J. Kozloski's current address: Biometaphorical Computing Group, IBM T. J. Watson Research Center, P.O. Box 218, Route 134, Yorktown Heights, NY 10598. Copyright (C) 2002 Society for Neuroscience $0270-6474 / 02 / 226290-12 \$ 15.00 / 0$ individual differences are readily detectable (Marvit and Crawford, 2000a).

The mormyrid fish ear consists of the sacculus and a gas-filled tympanic bladder (Stipetic, 1939; Fletcher and Crawford, 2001). The spherical bladder translates underwater sound pressure into the displacement that activates the hair cells (HCs) within the sacculus. The bladder is larger than the sacculus and is uniformly coupled to the sacculus over its entire extent. Thus, the auditory apparatus is much simpler than a cochlea, with a pulsating sphere driving the motion of the sacculus. This ear lacks the elongated sensory surface and basilar membrane that are important for peripheral frequency analysis in other vertebrates.

Despite the simplicity of the mormyrid ear, there are neurons in the CNS (midbrain) that are highly selective for click trains and tones of particular periods or frequencies. These neurons are suited to detecting features of grunts and moans (Crawford, 1993, 1997b). One physiological class is sensitive to click trains and is selective for particular interclick intervals common in grunts, whereas another is sharply selective for frequencies found in moans. It is likely that these represent the output of neural computations performed in the time domain (Crawford, 1993, 1997b; Kozloski and Crawford, 2000). These forms of feature selectivity could be produced by computational mechanisms that use synchronized spike trains as input, but to date, a detailed analysis of the primary afferent input to this system has not been available.

Here we present the first physiological analysis of the primary input to the mormyrid auditory system and show that complex receptive fields are an emergent property of the midbrain that does not exist in the primary afferent inputs to the brain. The 
primary afferents generate a faithful temporal representation of sounds through their synchronized spikes. This representation is relayed through the medulla and into the midbrain (Kozloski and Crawford, 1998, 2000), where it becomes transformed.

\section{MATERIALS AND METHODS}

Many of the methods used have been detailed previously (Crawford, 1993, 1997b; Kozloski and Crawford, 1998). The animal protocols were approved by the Institutional Animal Care and Use Committee of the University of Pennsylvania and comply with The Principles of Animal Care published by National Institutes of Health.

This study is based on the physiological characterization of 116 primary afferent auditory neurons. These neurons were recorded within the medulla because there is no peripheral portion of the nerve that can be exposed for recording; the sacculus is close to the brain surface, with auditory axons rapidly entering the medulla and coursing toward their midline targets (Kozloski and Crawford, 1998).

Single auditory axons were isolated on the basis of standard electrophysiological criteria, using dye-filled electrodes. Electrodes were advanced into the axon bundle in the lateral part of the medulla, distant from the medially positioned medullary nuclei. We used standard techniques to isolate single afferent neurons on the basis of spike waveform characteristics. One to four neurons were characterized per fish, all within $200 \mu \mathrm{m}$ of each other, in one electrode track. Only neurons that were within $200 \mu \mathrm{m}$ of the injection site were included. We then confirmed that the extracellular recordings were made within the axon bundle after iontophoresis of neurobiotin (Kawasaki and Guo, 1996; Kozloski and Crawford, 1998). We used a low injection current $(1 \mu \mathrm{A})$, short injection time $(2.5 \mathrm{~min})$, and short survival time $(10 \mathrm{~min})$ to prevent spread of neurobiotin beyond the axons local to the recording site. In most cases, only one to several axons were stained, confirming that the recordings were made in the nerve (Fig. 1). It was not necessary for our analyses to identify the particular axon from which each set of physiological data were obtained. If any label was detected within medullary auditory neurons, the corresponding physiology was eliminated from our sample because it was then possible that the primary afferents might have been secondarily labeled after uptake by first-order medullary neurons. An analysis of response latencies provided additional confirmation that our recordings were from primary afferents. Latency was measured as the delay between the peak of the click stimulus and the onset of the first evoked spike measured in the click peristimulus time histograms (PSTHs). The mean spike latency to a click stimulus was only $1.2 \mathrm{msec}$ $( \pm 0.6 \mathrm{SD})$, shorter than expected for higher-order medullary neurons.

Animals and preparation. Two sound-producing mormyrid species, Pollimyrus adspersus and Gnathonemus petersii (Crawford, 1997a), were imported from Nigeria by commercial dealers. More is known about the acoustic repertoire of $P$. adspersus, and the social settings in which the adult breeding fish use sounds, than for $G$. petersii. To date, studies of $G$. petersii have been based on juvenile fish and have revealed only simple clicking sounds used during agonistic behavior (Rigley and Marshall, 1973; Fletcher and Crawford, 2001). The acoustic repertoire of juvenile $P$. adspersus is similarly impoverished. It seems likely that adult $G$. petersii also use complex courtship sounds, but this matter awaits behavioral studies of breeding animals.

The ears of the two species appear nearly identical, both having a tympanic bladder coupled to the sacculus. The physiology of the primary afferents was also indistinguishable, on the basis of the distribution of characteristic frequencies (CFs) and synchronization coefficients $(p>$ 0.05; Mann-Whitney $U$ test). The data were combined for analysis and presentation. Any differences in the communication signals of these two species are more likely to be reflected in the physiology of higher-order neurons that extract features from the temporally encoded inputs. A total of 76 neurons from 25 Pollimyrus and 40 neurons from 16 Gnathonemus were used for this study. These fish were immobilized with an intramuscular injection of Flaxedil (gallamine triethiodide: $0.4 \mu \mathrm{g} / \mathrm{g}$ body weight) and placed on a Plexiglas platform. The fish were respirated with fresh, oxygenated water pumped through the gills. The CNS electric organ discharge motor command was used to monitor the animal's condition during the experiment. The command was recorded with a differential silver wire electrode placed beside the electric organ in the caudal peduncle. Experiments were terminated if the normal continuous volley of command signals $(\sim 10 / \mathrm{sec})$ became abnormal.

After a submucosal injection of the local anesthetic Lidocaine, the skin above the dorsal region of the skull was removed. A brass-retaining rod
A

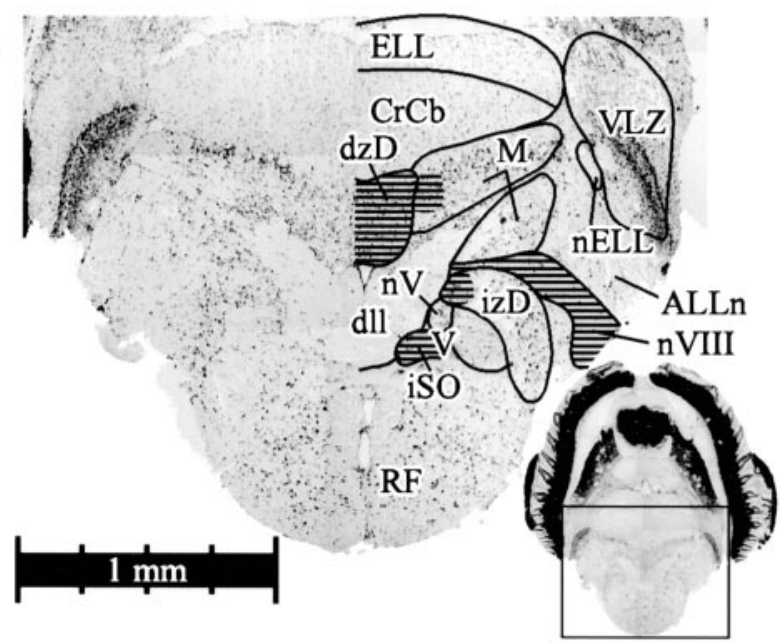

B

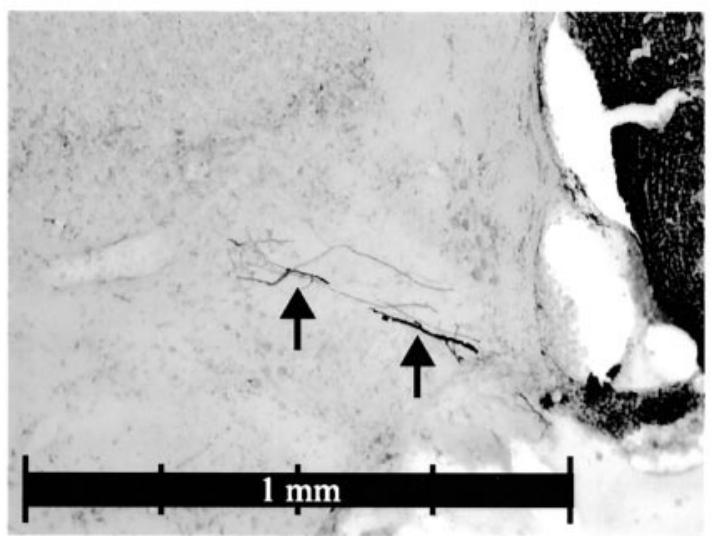

Figure 1. Transverse section of the mormyrid medulla $(A)$ and labeled axons in the saccular branch of the nVIIIth nerve $(B)$. In $A$, dashed-line areas highlight auditory regions. In $B$, arrows point to neurobiotin-filled afferent axons of the nerve. Note that only axons (fibers) are filled. These axons passed in and out of the section (60 $\mu \mathrm{m}$ thick). ALLn, Anterior lateral line nerve; $C r C b$, crista cerebellaris; $d l l$, decussation of the lateral lemniscus; $d z D$, dorsomedial zone of the descending octaval nucleus; $E L L$, electrosensory lateral line lobe; $i S O$, intermediate secondary octaval nucleus; $i z D$, intermediate zone of the descending octaval nucleus; $M$, medial octaval nucleus; $n E L L$, nucleus of electrosensory lateral line lobe; $n V$, nucleus of the descending tract of $\mathrm{V}$ (trigeminal); $n V I I I$, eighth cranial nerve; $R F$, reticular formation; $V L Z$, ventrolateral zone of ELL.

was cemented on to the anterior portion of the exposed skull and clamped to the platform to immobilize the head. A $3 \mathrm{~mm}$ access hole was drilled in the dorsal-caudal region of the skull exposing a small region of the brain surface for microelectrodes. A $5 \mathrm{~mm}$ plastic well was cemented to the skull and filled with Fluoroinert (3M FC-77; 3M, St. Paul, MN), an inert liquid that protected the brain by preventing water from entering the brain case. Finally, the platform was centered in a calibrated acoustic tank (Crawford, 1997b) and lowered until the fish's ears were submerged $25 \mathrm{~mm}$ below the surface of the water. Sounds were produced by an underwater speaker (University UW 30; University Sound, Buchanan, MI) placed at the bottom of the tank that rested on a Technical Manufacturing Corporation (Peabody, MA) vibration isolation table. This entire apparatus was enclosed in an Industrial Acoustics Corporation (New York, NY) sound-attenuating booth (400 series). The stimulus generation and data collection computer (Gateway 486 microcomputer) and associated hardware were housed outside of the booth.

Signal generation. Tone bursts and click trains were generated by a Gateway 486 processor microcomputer and digital to audio (D/A) hardware from Tucker Davis Technologies. Tones were produced with cosine on/off ramps of $30 \mathrm{msec}$. The stimulus signal was processed through a 

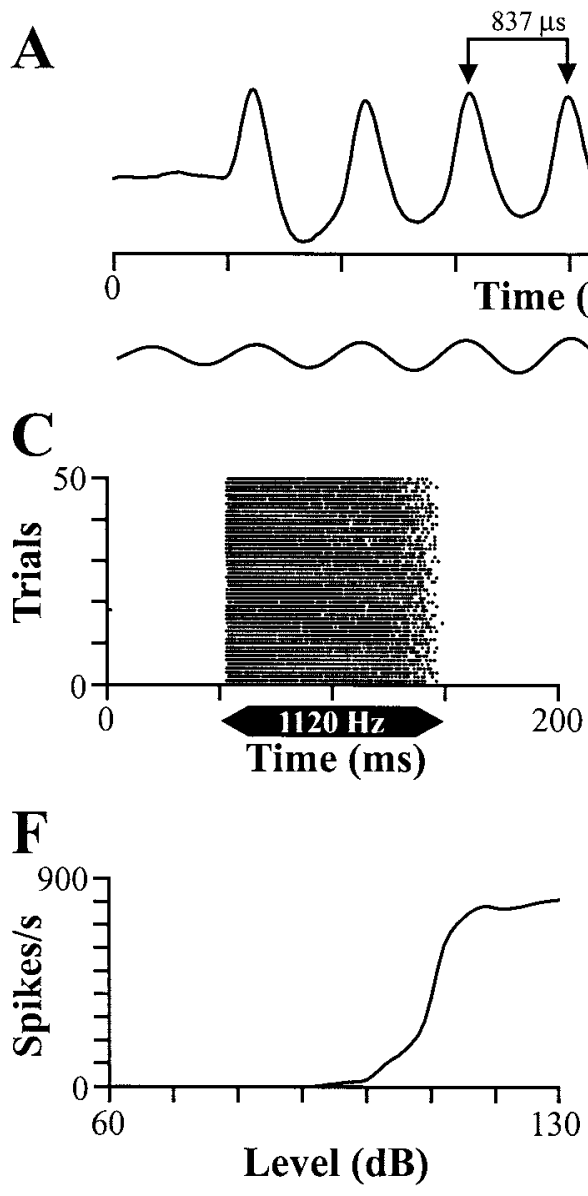

B

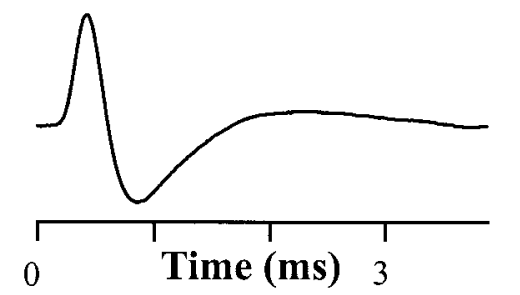

$\mathbf{E}$

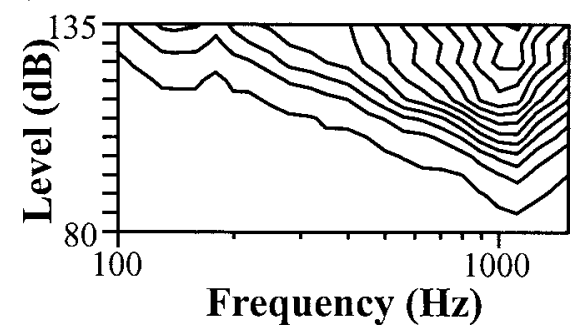

$\mathbf{H}$

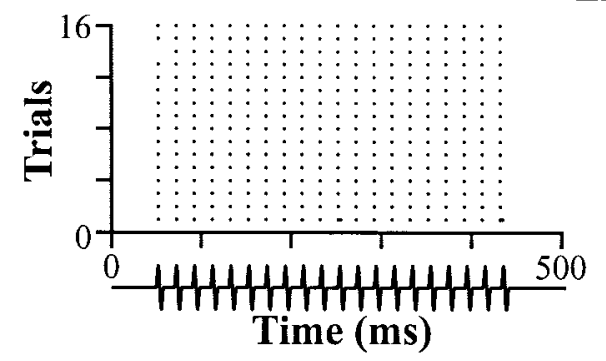

Figure 2. Example afferent response profile. A, Physiological trace recorded from an afferent with a juxtacellular glass pipette and stimulated with a tone $(1120 \mathrm{~Hz}, 125 \mathrm{~dB})$. Note the high degree of synchronization and firing rate equivalent to the stimulus frequency. The stimulus waveform is shown below the spike trace, as it ramps on (rise time $=30 \mathrm{msec}) . B$. Averaged physiological trace $(n=10)$ of the same neuron from $A$, stimulated with a lower-frequency tone $(300 \mathrm{~Hz}, 125 \mathrm{~dB})$. C , Peristimulus histogram for a $1120 \mathrm{~Hz}$ tone stimulus. $D$, Iso-level response function (125 dB). Arrow in $D$ indicates the failure frequency. $E$, Response area. Each line in $E$ represents an iso-response (firing rate) in increasing step sizes of 80 spikes per second. This neuron had no spontaneous activity, and the first contour line (lowest threshold) is 1 spike per second. $F$, Rate level function at $909 \mathrm{~Hz}$. Note that this neuron was synchronized to the tone period with a single spike, and thus response rate saturation was equivalent to stimulus frequency (909 $\mathrm{Hz}$ ) $G$, Synchronized spikes in response to click trains. H, Responses per train (left axis, solid line) and per click (right axis, dashed line) as a function of ICIs. $A-F$ were from an entrained type I neuron, and $G$ and $H$ were from an entrained type II neuron.

D/A converter, a Crown power amplifier, and a programmable attenuator. Acoustic stimuli generated by the speaker were recorded with a hydrophone (Bruel \& Kjaer 8103) at the location of the fish, for calibration and monitoring. Sound pressures are reported as $\mathrm{dB}$ rms re: $1 \mu \mathrm{Pa}$ for tones and $\mathrm{dB}$ peak re: $1 \mu \mathrm{Pa}$ for clicks. Sounds were presented up to $135 \mathrm{~dB}$ because this is the upper end of the natural range used by these animals (Crawford et al., 1986).

Single neuron extracellular physiology. Microelectrodes were made from glass pipettes pulled on a Flaming-Brown horizontal glass micropipette puller (P-97). These electrodes were backfilled with $2 \%$ neurobiotin (Sigma, St. Louis, MO) in $0.5 \mathrm{M} \mathrm{KCl}$. A silver chloride wire was inserted into the electrode, and the tip was beveled (micropipette beveler, BV-10; Sutter Instrument Co.) until the impedance of the electrode was 15-30 $\mathrm{M} \Omega$. The electrode was mounted on a Burleigh Inchworm microdrive (IW-711-01) and micromanipulator. The electrode was manually positioned onto the dorsal surface of the brain in reference to brain surface landmarks. The electrode was then advanced from near the midline, along a track $30^{\circ}$ off vertical, to intersect the auditory nerve close to the lateral border of the brain.

The physiological signal was preamplified (gain $=1000)$ by a batterypowered amplifier (DAM 50; World Precision Instruments, Sarasota, FL) located inside the booth. The amplified recording was filtered (KrohnHite filter; Model 3100A) and displayed on the oscilloscope (Tektronix, 2221A). This signal was sent to a hoop discriminator (Tucker Davis Technologies) to isolate and time stamp the action potentials with a resolution of $1 \mu \mathrm{sec}$. A custom computer program coordinated the stimulus presentation and data collection.

Auditory neurons were isolated by searching for responses while presenting acoustic stimuli that were composed of a series of tone bursts and click trains. Response areas for these neurons were mapped by recording responses (spikes) to tones for a range of frequencies $(100-2500 \mathrm{~Hz} ; 0.08$ $\log _{10} \mathrm{~Hz}$ steps) and at different levels $(50-135 \mathrm{~dB}$ re: $1 \mu \mathrm{Pa} ; 3-5 \mathrm{~dB}$ steps). The threshold for an excitatory response was defined as the lowest level that evoked a spike rate (SR) at least 2 SDs above the spontaneous spike rate for the neuron (if $S R=0$, response criterion $\geq 1$ spike per second). The CF was determined as the frequency that had the lowest threshold in the response area; the threshold at the CF is called the best sensitivity (BS). Frequency tuning curves (FTCs) were also plotted by connecting the threshold at each frequency. The degree of tuning $\left(\mathrm{Q}_{10} \mathrm{~dB}\right)$ for each neuron was estimated by dividing the $\mathrm{CF}$ by the frequency bandwidth of the FTC at $10 \mathrm{~dB}$ above BS. Additionally, the bandwidth $\left(\mathrm{BW}_{125 \mathrm{~dB}}\right)$ of the FTC was used to characterize each neuron during suprathreshold stimulation (measured in log units). Iso-level functions were also graphed, in which the effects of frequency were observed at a constant, suprathreshold level $(125 \mathrm{~dB})$. The frequency that evoked the maximal firing rate (MR) at $125 \mathrm{~dB}$ was called the best frequency $(\mathrm{BF})$. The relationship between intensity and spike rate, the rate-level function (RLF), was obtained by plotting responses to a tone of a particular frequency (e.g., CF) over an increasing series of levels (5 dB steps). 

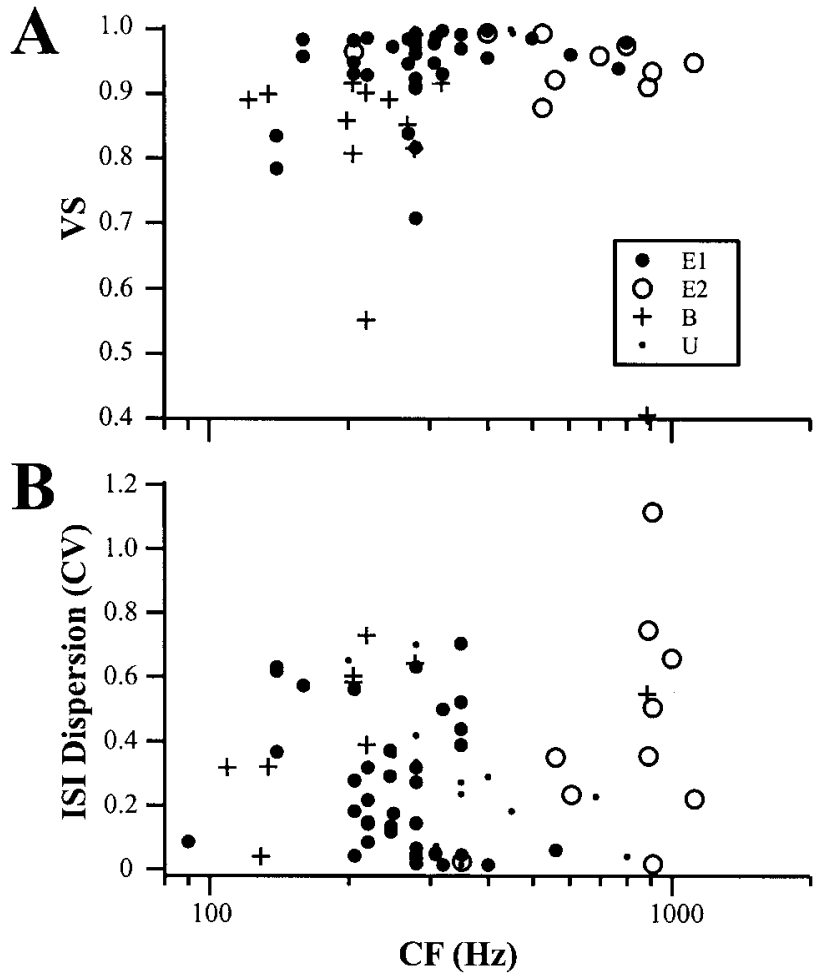

Figure 3. Distribution of synchronization $(A)$ and ISI dispersion $(B)$ over the range of characteristic frequencies. Each synchronization and ISI dispersion value was taken at either the $\mathrm{CF}$ or $\mathrm{BF}$ of the neuron at $125 \mathrm{~dB}$, at least $10 \mathrm{~dB}$ over threshold. Inset provides a key to the symbols. E1, Entrained type I; E2, entrained type II; $B$, burster; $U$, unclassified.

Table 1. Principal components analysis

\begin{tabular}{|c|c|c|c|c|}
\hline & Factor 1 & Factor 2 & Factor 3 & Factor 4 \\
\hline \multicolumn{5}{|l|}{ Variable } \\
\hline SR & -0.258 & $0.764 *$ & 0.129 & -0.092 \\
\hline $\mathrm{BF}$ & $0.852 *$ & -0.102 & 0.164 & 0.209 \\
\hline MR & $0.901 *$ & 0.242 & -0.011 & -0.160 \\
\hline VS & 0.032 & -0.434 & -0.574 & -0.511 \\
\hline $\mathrm{CV}$ & -0.134 & -0.154 & -0.605 & $0.719 *$ \\
\hline $\mathrm{CF}$ & 0.598 & -0.229 & 0.437 & 0.323 \\
\hline BS & -0.610 & -0.607 & 0.315 & 0.003 \\
\hline $\mathrm{BW}_{125}$ & $0.807 *$ & 0.174 & -0.390 & -0.004 \\
\hline RLF slope & 0.665 & -0.476 & 0.130 & -0.201 \\
\hline Eigen values & 3.448 & 1.541 & 1.198 & 1.00 \\
\hline$\%$ of total variance & 38.3 & 17.1 & 13.3 & 11.1 \\
\hline
\end{tabular}

Bold values with an asterisk identify loadings with magnitude $>0.7$.

To visualize temporal responses to tones, PSTHs were constructed by presenting 50 tone bursts at a particular frequency, level, and starting phase. The PSTH displays the probability of a spike occurring within a given time bin during the course of a stimulus presentation. To measure how well action potentials synchronized to the tone frequency (i.e., the degree of phase locking), the VS was calculated from a PSTH by converting spike times to unit vectors and computing the average vector length (Goldberg and Brown, 1969). VS values range from 1.0 (perfect synchrony) to 0.0 (no synchrony). The Rayleigh test was used to determine whether the angular distribution of VS was significantly different from random (Batschelet, 1981). Interspike interval histograms (ISIHs) were also examined, and interval dispersion was quantified with the $\mathrm{CV}$ $(\mathrm{CV}=\mathrm{SD} /$ mean interval $)$.

Responses to 400 msec click trains were also analyzed. Click trains were composed of broadband pulses $(160-3000 \mathrm{~Hz})$ with constant ICIs

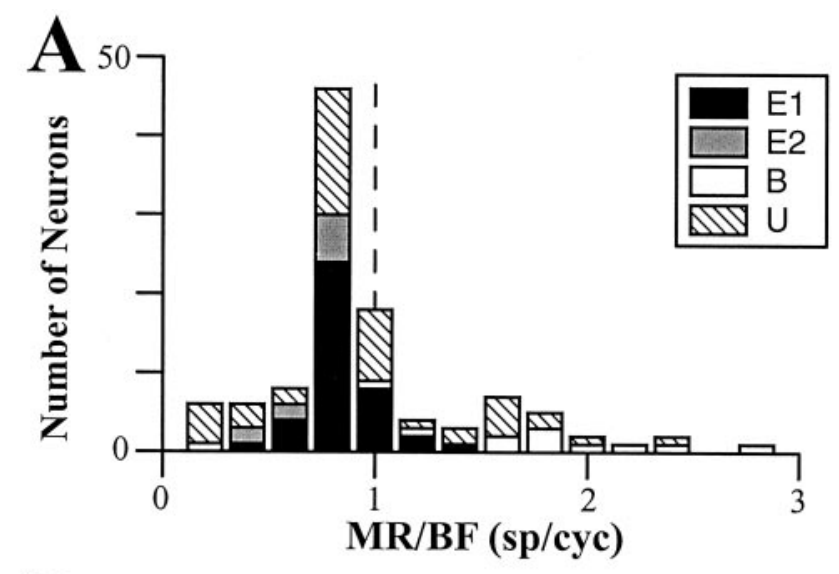

B

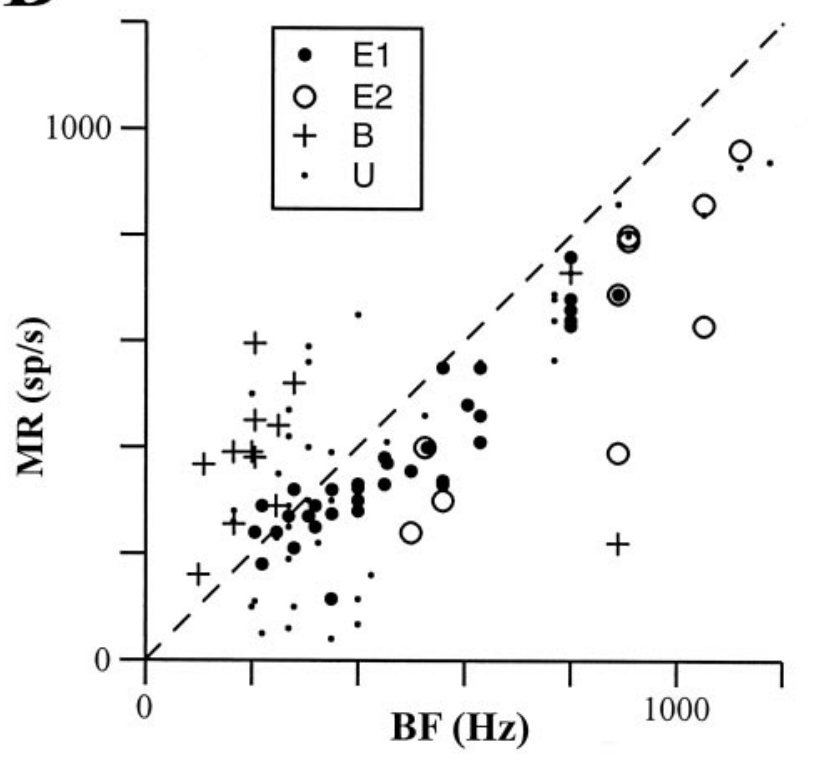

Figure 4. Distribution of entrainment performance as measured by the ratio of maximum rate to best frequency $(M R / B F)$. $A$, Frequency distribution of ratios. The dashed line corresponds to the bin where the maximum rate matches the best frequency, i.e., a single spike per cycle or perfect entrainment. Each bin is 0.2 units wide. Afferents that fell in the center of the distribution were considered to be entrained. $B$, Plot of best frequency relative to the maximum rate. The dashed line corresponds to the line where the maximum rate matches the best frequency, i.e., one spike per cycle or perfect entrainment. E1, Entrained type I; E2, entrained type II; $B$, burster; $U$, unclassified.

that ranged from 6 to $100 \mathrm{msec}$. The dependence of spike rate on ICIs was examined by plotting the number of spikes evoked as a function of ICIs. PSTHs and ISIHs were also used to characterize temporal responses to click trains. All-order ISIHs, formally equivalent to autocorrelation functions, were constructed to reveal any correspondence of the predominant interval in the spike train and the stimulus ICI (Licklider, 1951; Perkel et al., 1967; Moller, 1970). These were plotted by computing intervals between a given spike and all spikes within the spike train.

Tissue processing. After physiology, each fish was anesthetized with MS-222 and perfused with heparinized physiological saline (PBS) and phosphate-buffered $(1.25 \%)$ paraformaldehyde/(1.25\%) glutaraldehyde fixative. The brain was removed, postfixed, embedded in gelatin, and prepared for frozen sectioning.

Transverse sections $(60 \mu \mathrm{m})$ were washed in PBS, bathed in $0.5 \%$ $\mathrm{H}_{2} \mathrm{O}_{2}$, and then incubated overnight in a solution containing avidinconjugated horseradish peroxidase $(0.3 \%$ Triton X-100, PBS; Vector Laboratories, Burlingame, CA). Sections were then soaked in phosphatebuffered diaminobenzidine (Sigma) containing $\mathrm{H}_{2} \mathrm{O}_{2}$ and $0.04 \%$ ammonium sulfate to visualize the neurobiotin-chromagen conjugate (Ka- 

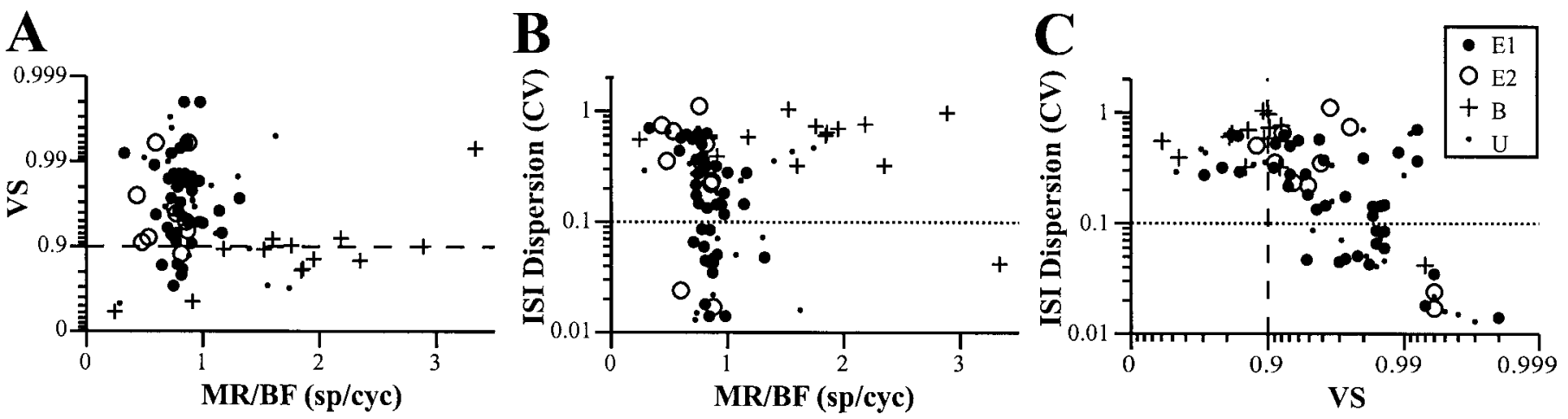

Figure 5. Scatter plots for synchronization (VS), ISI dispersion $(C V)$, and MR/BF ratio. Synchronization (VS) was plotted as a function of MR/BF ratio $(A)$, and ISI dispersion was plotted as a function of MR/BF $(B)$ and as a function of synchronization $(C)$. The dashed line represents a criterion for which neurons were considered highly synchronized $(A, C)$, and the dotted line represents a criterion for low dispersion $(B, C)$ (Young et al., 1988; Joris et al., 1994). Synchronization was plotted on a reverse log axis to disperse data with high vector strengths $(A, C)$ (Joris et al., 1994). E1, Entrained type I; E2, entrained type II; $B$, burster; $U$, unclassified.

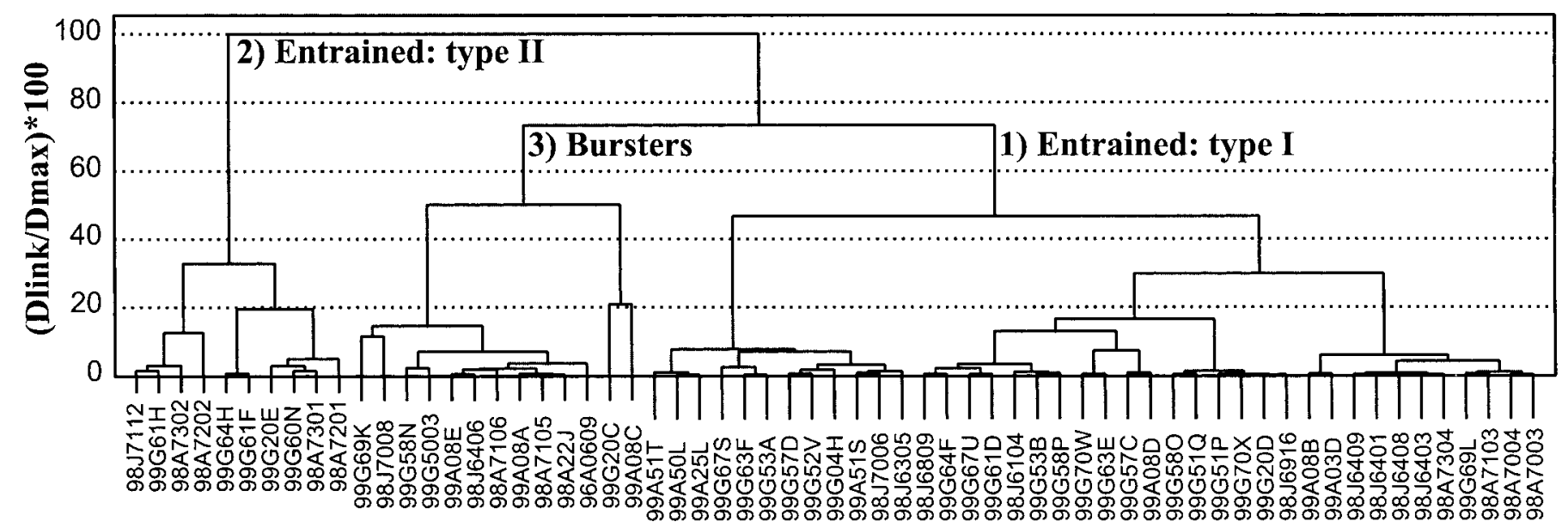

Figure 6. Cluster analysis of physiological variables. The analysis was performed using the variables SR, MR/BF, CV, CF, BS, BW 125 , and RLF slope.

wasaki and Guo, 1996; Kozloski and Crawford, 1998). The mounted sections were counterstained with cresyl violet.

Statistical analyses. Principle component analysis (see Table 1), descriptive statistics (see Table 2), nonparametric statistics (Mann-Whitney $U$ and Wilcoxon signed rank tests), and cluster analysis (CA) were calculated with Statistica v 2.0 (StatSoft). The multidimensional data set (nine variables) was explored using principal components analysis (PCA) to examine the contributions of each physiological variable to those PCA factors capturing most of the variance in the data (factors 1-4). A CA was then performed to identify natural clustering (i.e., classes) among the neurons sampled. After standardizing the data, CA was performed using squared Euclidean distances and Ward's method linkage rules. These analyses (PCA and CA) were based on SR, MR, BF, CV, CF, BS, BW $125 \mathrm{~dB}$, and RLF slope. $\mathrm{Q}_{10 \mathrm{~dB}}$ was excluded from these analyses because it is so closely related to two of the variables that were included (i.e., $\mathrm{BW}_{125 \mathrm{~dB}}$ and $\mathrm{CF}$ ). In cases in which multiple comparisons were made with the Mann-Whitney $U$ and Wilcoxon tests, appropriate adjustments of the $p$ values were made using Bonferroni correction (Howell, 1992).

\section{RESULTS}

The responses of mormyrid primary auditory afferents were markedly different from those recorded more centrally in this auditory system. The primary afferents produced sustained responses to tones, with spikes strongly entrained to the temporal structure of the acoustic stimulus (Fig. 2). Cycle-by-cycle stimulus following was observed at rates as high as $1.12 \mathrm{kHz}$ in some afferents, with essentially no spike rate adaptation (Fig. 2C,D). In contrast, tones elicited highly phasic responses in midbrain neu- rons, with poorer synchrony (i.e., lower VS and higher $\mathrm{CV}$ ). Sharp onset responses were often followed by suppression of spike rate below the spontaneous rate and rebound-excitation at stimulus offset. Unlike the afferents, the rate-level functions of midbrain neurons were frequently nonmonotonic (Crawford, 1993, 1997b).

The majority of primary afferents produced a single synchronized spike on almost every stimulus cycle, thus entraining to the stimulus [i.e., $80 \%$ had VS $>0.9$ and $77 \%$ had $\mathrm{CV}<0.5$; criteria for entrainment adopted from Joris et al. (1994)]. The median VS was high $(0.96$ at $125 \mathrm{~dB})$ (Fig. $3 A)$, and the median $\mathrm{CV}$ was low $(0.28$ at $125 \mathrm{~dB})$ (Fig. $3 B)$, corresponding to narrow, unimodal, ISI distributions. It is possible to obtain moderately low CVs without entrainment [e.g., in chopper neurons (Kozloski and Crawford, 2000)], but this was never observed in primary afferents.

The spontaneous activity of afferents ranged widely, from 0 to 400 spikes per second, but most $(67 \%)$ had some spontaneous activity. Afferents with spontaneous activity (SR $>1$ spike per second) were $\sim 10 \mathrm{~dB}$ more sensitive (i.e., lower thresholds) than those without (median threshold: 100.0 vs $109.1 \mathrm{~dB} ; \mathrm{U}=852 ; n=$ 51,57; $p<0.001)$.

In addition to entrained afferents, we also observed a smaller number of afferents that produced bursts of spikes on each 

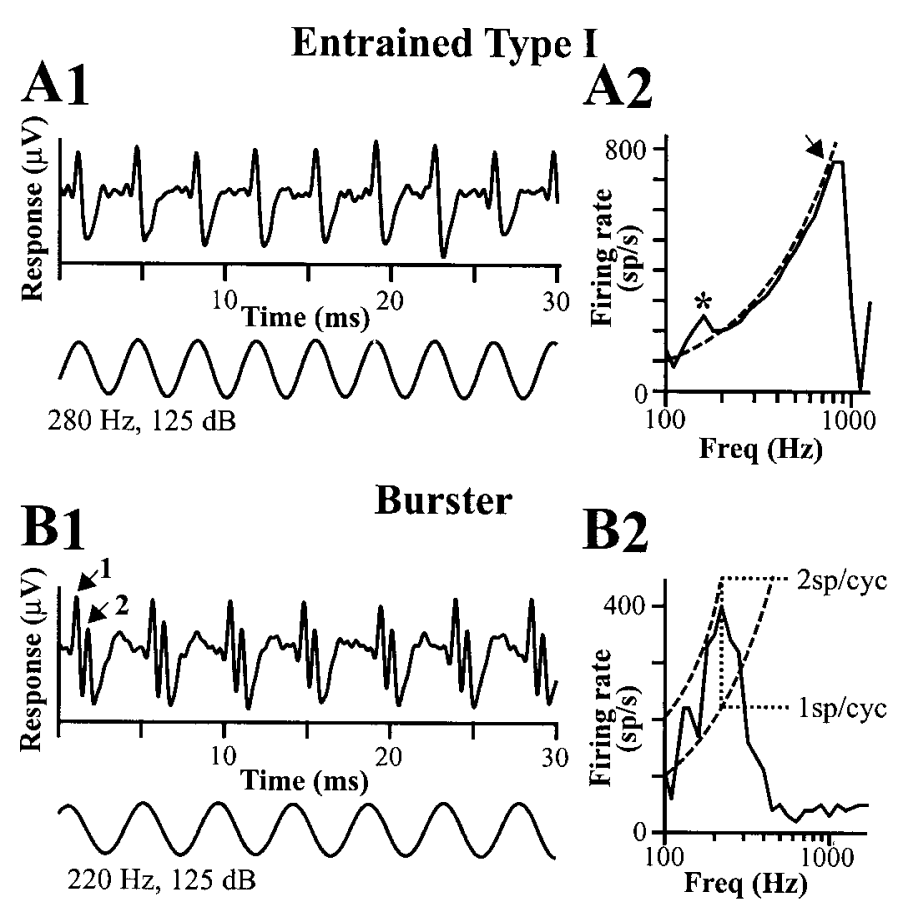

Figure 7. Extracellular spike waveforms $(A 1, B 1)$ and iso-level functions $(A 2, B 2)$ for neurons stimulated with tones at $125 \mathrm{~dB} . A 1, A 2$, Entrained neuron (type I). B1, B2, Burster neuron. Arrows $(1,2)$ point to each of the two spikes in the burster response (B1). Dashed lines in iso-level functions plot perfect entrainment, or one spike per cycle. Entrained neurons showed nearly perfect entrainment (A2). The asterisk indicates bursting at low frequencies $(<200 \mathrm{~Hz})$, and the arrow points to the failure frequency of the entrained neuron $(A 2)$. For the burster iso-level function (B2), an additional dashed line plots the two spike per cycle (bursting) trajectory. Dotted lines show the $\mathrm{BF}(220 \mathrm{~Hz})$ response relative to the 1 and 2 spikes per cycle trajectories.

stimulus cycle. This bursting degraded entrainment by increasing the $\mathrm{CV}$ of the ISI distribution and by reducing VS. The bursting afferents also had low-frequency CFs near $200 \mathrm{~Hz}$ and relatively symmetrical response areas, compared with the entrained afferents. These observations suggested that there might be two or more physiological classes within our afferent sample.

\section{Characteristics of afferent response clusters}

We examined the afferent sample using our physiological variables to determine whether there were distinct physiological classes. Principal components analysis of the multidimensional data set showed that $80 \%$ of the variance was captured by the first four factors, derived from nine physiological variables (Table 1). We used the loading of our physiological variables to identify those contributing most heavily to these four factors. Among the important variables were the maximum firing rate, characteristic frequency, best excitatory frequency, ISI dispersion, and vector strength. Nevertheless, two-dimensional analyses of these variables failed to reveal distinct classes (Figs. 3-5). We pursued this analysis further with a multidimensional cluster analysis.

The CA, based on nine physiological variables (Table 1), indicated that the sample consisted of three major clusters, one corresponding to bursting afferents and two others corresponding to entrained afferents (Fig. 6). The largest cluster (entrained type I: $35 \%$ ) was composed of afferents that strongly entrained to a wide range of stimulus frequencies (Figs. 2D, 7A). A synchronized spike was generated for each stimulus cycle, except at the lowest frequencies $(<200 \mathrm{~Hz})$ where synchronized bursts were sometimes produced (Fig. 7A2). In an iso-level frequency function, the response (spikes per second) was essentially identical to a plot of the number of stimulus cycles as a function of tone frequency. Thus, these afferents exhibited high-fidelity frequency following (Fig. 7A2, dashed line). As frequency was increased, an upper limit for entrainment was reached, the failure frequency, and the response rate dropped precipitously beyond this point (Fig. 7A2). Failure frequencies were as high as $890 \mathrm{~Hz}$ for type I afferents. Consequently, the frequency-band of entrainment encompassed nearly the entire audibility range, as measured behaviorally (Marvit and Crawford, 2000a). Near threshold, these neurons responded best at lower frequencies, with CFs concentrated at $\sim 265 \mathrm{~Hz}$ (CF dispersion: $25-75 \%$ quartile $=217-308 \mathrm{~Hz}$ ).

The second cluster of entrained afferents (type II: 15.9\%) was quite similar to type I with respect to degree of tuning and entrainment. However, this second cluster was distinguished by higher CFs and BFs (median CF, 900 vs 265 Hz; median BF, 900 vs $400 \mathrm{~Hz}$ ). These type II neurons also had lower spontaneous rates (median $\mathrm{SR}=0$ vs 5.2 spikes per second) and steeper rate level functions (median slope $=31.1$ vs 11.6 spikes per second per decibel). The high BFs in this cluster meant that these afferents entrained at even higher frequencies, some $>1.0 \mathrm{kHz}$ (Fig. 2C, Table 2).

The third cluster revealed by the CA corresponded to the bursting afferents mentioned above. The afferents in this cluster fired bursts of spikes on every stimulus cycle for stimuli within the response area (Fig. 7B1). The frequency range of stimulus following was also comparatively restricted (Fig. 7B2), and the response areas were more $\mathrm{V}$-shaped than those of other afferents (Fig. 8B1). The bursting resulted in broader period histograms, with a second mode in the ISIH corresponding to the intervals between the spikes within each burst (Fig. 9B). The CFs were also restricted to a narrow, low-frequency range (CF dispersion: $25-$ $75 \%$ quartile range $=135-220 \mathrm{~Hz}$ ) similar to that of the entrained type I afferents.

\section{Intensity coding and thresholds}

Although all primary afferents had monotonic RLFs, there were clear differences in RLFs between the afferent clusters identified above. The entrained afferents (type I and II) had steep, rapidly saturating, rate-level functions with near-perfect synchrony achieved within $\sim 10 \mathrm{~dB}$ of rate threshold (Fig. 8A2,A3). In contrast, the RLFs of bursting afferents were shallow and only began to saturate at the highest levels (Fig. 8B2).

These contrasts in rate-level functions yielded differences in the RLF slopes within the dynamic range (DR) (dB range from 20 to $80 \%$ of maximum response) (Fig. 8B2). The bursting afferents had the shallowest slopes (median $=9.8$ spikes per second per decibel) as compared with the steeper slopes of the entrained type I (median $=11.6$ spikes per second per decibel) and type II afferents (median = 31.1 spikes per second per decibel). The slopes of type II afferents were significantly steeper than both bursting $(\mathrm{U}=76 ; p<0.003)$ and the type I afferents ( $\mathrm{U}=22 ; p<0.008)$.

Synchronization increased as stimulus intensity increased, with entrained (type I) and bursting afferents showing significant synchronization at levels $\sim 8 \mathrm{~dB}$ below the rate-based threshold (Fig. $8 A 3, B 3)$. Because type II neurons usually had little spontaneous activity, synchronization could not be measured below rate threshold.

For the entrained type I neurons, the median difference between synchronization and rate thresholds was $10.5 \mathrm{~dB}(p<0.05$; 
Table 2. Summary statistics for the physiology of the entire afferent sample and the three clusters (see Fig. 6)

\begin{tabular}{|c|c|c|c|c|c|c|c|c|c|c|c|}
\hline Quartile & $\begin{array}{l}\text { SR (spikes } \\
\text { per second) }\end{array}$ & $\mathrm{CF}(\mathrm{Hz})$ & $\mathrm{BS}(\mathrm{dB})$ & $\mathrm{Q}_{10 \mathrm{~dB}}$ & $\mathrm{BW}_{125}$ & $\begin{array}{l}\text { MR (spikes } \\
\text { per second) }\end{array}$ & $\mathrm{BF}(\mathrm{Hz})$ & $\begin{array}{l}\text { MR/BF } \\
\text { (spikes } \\
\text { per cycle) }\end{array}$ & VS & $\mathrm{CV}$ & $\begin{array}{l}\text { RLF } \\
\text { slope }\end{array}$ \\
\hline \multicolumn{12}{|l|}{ All afferents } \\
\hline$(\min ) 0 \%$ & 0.0 & 90 & 65.0 & 0.28 & 0.03 & 40.0 & 100 & 0.11 & 0.41 & 0.01 & 1.2 \\
\hline $25 \%$ & 0.0 & 220 & 95.0 & 1.02 & 0.35 & 262.5 & 270 & 0.75 & 0.91 & 0.07 & 7.8 \\
\hline (median) $50 \%$ & 10.1 & 280 & 105.0 & 1.66 & 0.62 & 352.5 & 351 & 0.86 & 0.96 & 0.28 & 12.1 \\
\hline $75 \%$ & 102.9 & 350 & 115.0 & 2.59 & 0.93 & 557.5 & 630 & 1.00 & 0.99 & 0.52 & 19.9 \\
\hline$(\max ) 100 \%$ & 434.1 & 1120 & 135.0 & 9.33 & 1.28 & 962.5 & 1176 & 3.34 & 1.00 & 1.11 & 58.9 \\
\hline$n$ & 116 & 86 & 85 & 67 & 72 & 110 & 110 & 110 & 91 & 90 & 107 \\
\hline \multicolumn{12}{|l|}{ Entrained: type I } \\
\hline$(\min ) 0 \%$ & 0.0 & 90 & 65.0 & 0.28 & 0.11 & 115.0 & 206 & 0.33 & 0.71 & 0.01 & 2.5 \\
\hline $25 \%$ & 0.0 & 217 & 96.5 & 1.17 & 0.28 & 270.0 & 317 & 0.75 & 0.93 & 0.06 & 6.9 \\
\hline (median) $50 \%$ & 5.2 & 265 & 109.0 & 2.06 & 0.54 & 326.5 & 400 & 0.81 & 0.97 & 0.18 & 11.6 \\
\hline $75 \%$ & 73.5 & 308 & 112.8 & 2.90 & 0.89 & 422.5 & 572 & 0.91 & 0.99 & 0.38 & 17.4 \\
\hline$(\max ) 100 \%$ & 251.2 & 560 & 125.0 & 9.33 & 1.28 & 760.0 & 890 & 1.32 & 1.00 & 0.70 & 25.7 \\
\hline$n$ & 40 & 40 & 40 & 36 & 40 & 40 & 40 & 40 & 40 & 40 & 40 \\
\hline \multicolumn{12}{|l|}{ Entrained: type II } \\
\hline$(\min ) 0 \%$ & 0.0 & 351 & 70.0 & 0.48 & 0.25 & 240.0 & 500 & 0.44 & 0.88 & 0.02 & 8.9 \\
\hline $25 \%$ & 0.0 & 677 & 90.0 & 0.89 & 0.82 & 392.5 & 643 & 0.55 & 0.93 & 0.22 & 12.9 \\
\hline (median) $50 \%$ & 0.0 & 900 & 100.0 & 1.17 & 0.93 & 660.0 & 900 & 0.77 & 0.95 & 0.35 & 31.1 \\
\hline $75 \%$ & 2.0 & 909 & 113.5 & 1.23 & 1.02 & 797.5 & 1016 & 0.85 & 0.97 & 0.62 & 50.7 \\
\hline$(\max ) 100 \%$ & 91.7 & 1120 & 120.0 & 3.03 & 1.27 & 962.5 & 1120 & 0.88 & 0.99 & 1.11 & 58.9 \\
\hline$n$ & 10 & 10 & 10 & 10 & 10 & 10 & 10 & 10 & 10 & 10 & 10 \\
\hline \multicolumn{12}{|l|}{ Burster } \\
\hline$(\min ) 0 \%$ & 0.0 & 110 & 75.0 & 1.01 & 0.15 & 160.0 & 100 & 0.25 & 0.41 & 0.04 & 7.2 \\
\hline $25 \%$ & 2.0 & 135 & 95.0 & 1.23 & 0.51 & 290.0 & 167 & 1.53 & 0.82 & 0.39 & 9.0 \\
\hline (median) $50 \%$ & 97.3 & 206 & 97.0 & 1.57 & 0.60 & 390.0 & 206 & 1.84 & 0.89 & 0.61 & 9.8 \\
\hline $75 \%$ & 110.5 & 220 & 103.0 & 2.34 & 0.66 & 450.0 & 250 & 2.18 & 0.90 & 0.73 & 11.8 \\
\hline$(\max ) 100 \%$ & 287.0 & 890 & 115.0 & 4.40 & 1.16 & 730.0 & 890 & 3.34 & 0.99 & 1.04 & 26.8 \\
\hline$n$ & 13 & 13 & 13 & 10 & 13 & 13 & 13 & 13 & 13 & 13 & 13 \\
\hline
\end{tabular}

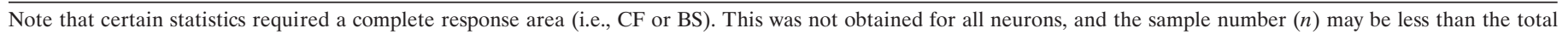
number of neurons.

Wilcoxon signed rank test). These afferents showed strong synchronization to tones at rate threshold (median VS at rate threshold $=0.75)$, and synchrony increased modestly as level was increased further (median VS at $125 \mathrm{~dB}=0.93$ ). Although the median level at which synchrony became saturated $($ median $=$ $95.7 \mathrm{~dB} ; 90 \%$ of maximal synchrony) was lower than the level at which rate became saturated (median $=103.5 \mathrm{~dB} ; 90 \%$ of maximal firing rate), the difference was not significant for entrained type I afferents $(p<0.13$; Wilcoxon signed rank test) (Fig. 8A3).

For the bursting afferents, the median difference between synchronization and rate thresholds was $12.0 \mathrm{~dB}$ ( $p=0.07$; Wilcoxon signed rank test). The bursters were more weakly synchronized at rate threshold (median VS at rate threshold $=0.5$; median threshold $=97 \mathrm{~dB}$ ) and improved their synchrony more slowly with increasing level (median VS at $25 \mathrm{~dB}=0.8$ ). The bursting afferents reached synchrony saturation before rate saturation (median level at synchrony saturation $=98.8 \mathrm{~dB}$ and median level at rate saturation $=119.7 \mathrm{~dB} ; p=0.07$; Wilcoxon signed ranked test) (Fig. 8B3).

\section{Maximum spike rates, synchronization, and ISI dispersion}

The data on maximum driven rates (MR), BF, ISI dispersion (CV), and synchronization (VS) have been plotted with separate symbols (Figs. 4, 5) for each of the clusters identified in the CA
(Fig. 6). The ratio of MR to BF was near unity for the entrained afferents because of their one spike per cycle firing behavior (Fig. 4). The MR/BF ratio was closer to 2 for the bursting afferents (Fig. 4A), and their MFs were clustered near $200 \mathrm{~Hz}$ (Fig. 4B). Note that the $\mathrm{MR} / \mathrm{BF}$ ratio for many of the entrained neurons was slightly $<1.0$ because of the on and off ramps of the tone bursts; the ratio was very close to 1 during the steady-state part of the tone. Similarly, the MR/BF ratio was usually just under 2 for the bursting afferents. It should also be pointed out that there was a very small $(12 \%)$, but statistically significant, difference in the $\mathrm{MR} / \mathrm{BF}$ ratio between the two species used in our studies $(G$. petersii and $P$. adspersus), with the ratio being smaller for $P$. adspersus.

The synchronization of bursting afferents to BF tones (125 dB) was significantly less (median VS $=0.89$ ) than the entrained afferents (type I: median VS $=0.97, \mathrm{U}=85, p<0.001$; type II: median VS $=0.95, \mathrm{U}=16, p<0.003$ ) (Fig. 5A). The ISI dispersion was significantly less among type I entrained afferents (median $\mathrm{CV}=0.18$ ) compared with the bursters (median $\mathrm{CV}=$ $0.61 ; \mathrm{U}=85 ; p<0.0003)$. Type II afferents had a median $\mathrm{CV}$ of 0.35 and were not significantly different from type I afferents $(\mathrm{U}=143 ; p=0.17)$ and bursters $(\mathrm{U}=43 ; p=0.17)$ (Fig. 5, Table 2). There was a significant negative correlation between $\mathrm{CV}$ and VS for the afferent sample as a whole $(r=0.67 ; p<0.05)$, reflecting the decline in interval dispersion as synchrony in- 

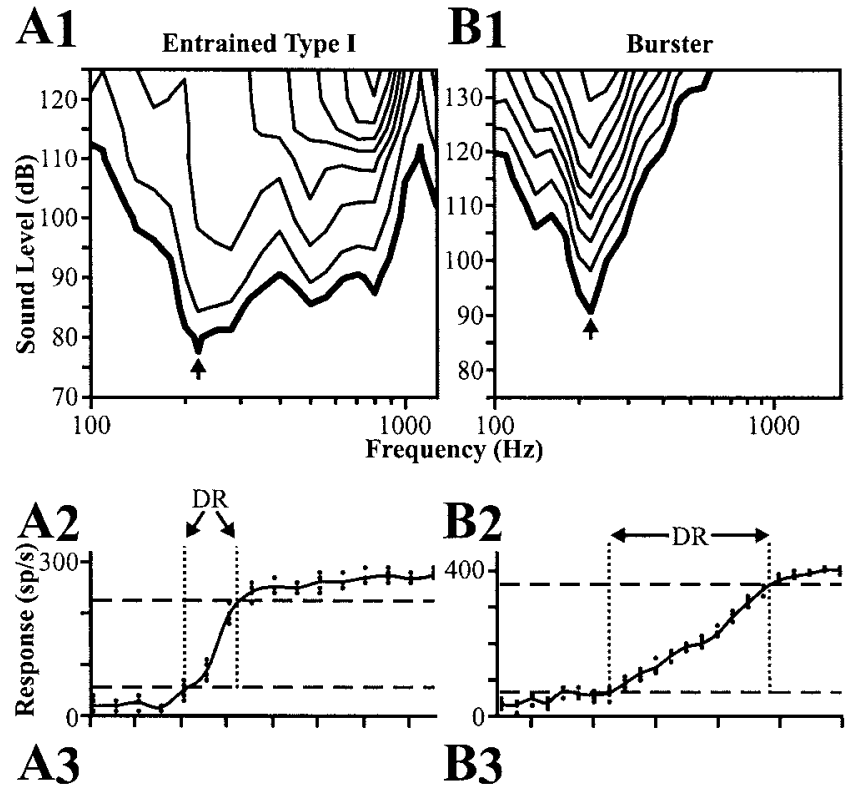

B3

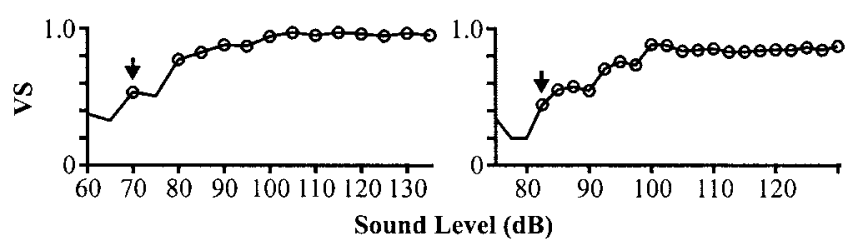

Figure 8. Response areas $(A 1, B 1)$, rate-level functions $(A 2, B 2)$, and synchronization level functions $(A 3, B 3)$ for the entrained type $\mathrm{I}$ and bursting afferents. The heavy solid contour line in each response area $(A 1$, $B 1)$ is the FTC, corresponding to a firing rate of 46 and 66 spikes per second, respectively. The other contour lines indicate iso-response curves, lines indicating where the stimulus parameters (frequency and level) elicit equal firing rates. Contour lines are in increasing step sizes of 100 and 50 spikes per second for $A 1$ and $B 1$, respectively. In each response area, arrows point to the $\mathrm{CF}$. In the rate-level functions in $A 2$ and $B 2$, the bottom dashed lines indicate the criterion response rate for threshold estimates, and the left-most dotted lines intersect the response curve indicating the threshold level. The DR was estimated by measuring differences between the threshold level and the level (right-most dotted line) that produced the $90 \%$ maximal response (top dashed line). Synchronization $(V S)$ was plotted as a function of level $(A 3, B 3)$, where circles indicate statistically significant synchronization (Rayleigh test; $p>0.9$ ), with arrows pointing to the synchronization threshold.

creased, and there was extensive overlap between the clusters in the $\mathrm{CV}$ versus VS plot (Fig. $5 C$ ).

\section{Tone sensitivity and the audiogram}

The response areas of the afferent population spanned both the amplitude spectrum of the vocalizations made by Pollimyrus and the audiogram (Fig. 10). Although CFs were widely distributed between $100 \mathrm{~Hz}$ and $1.0 \mathrm{kHz}$ (Table 2), half of the CFs were clustered between 200 and $300 \mathrm{~Hz}$, where the fundamental frequency of one of the key components of the courtship display lies (moan $F_{0}=250 \mathrm{~Hz}$ ) (Crawford et al., 1997a).

To compare our neurophysiological data with the behavior, we constructed FTCs for the afferents and extracted a neural threshold sensitivity curve from these tuning curves. At each frequency, a number of FTCs overlapped, each contributing one threshold to our estimate of the neural threshold sensitivity for that frequency. The threshold corresponding to the 90th percentile was found for each of the frequencies. Thus, at each frequency $10 \%$ of the thresholds fell below this threshold (i.e., were more sensitive), and $90 \%$ fell above. These 90th percentile thresholds (decibels) were used to draw the neural threshold curve (Fig. 10). The neural threshold curve showed a sensitivity maximum near the $250 \mathrm{~Hz}$ fundamental of the courtship sounds. This curve closely matched the Pollimyrus audiogram measured behaviorally (McCormick and Popper, 1984; Marvit and Crawford, 2000b; Fletcher and Crawford, 2001).

\section{Encoding single clicks and click trains}

Clicks were encoded with either a single short latency spike or a burst of spikes. Afferents without spontaneous activity $(<1$ spike per second) responded with one or two spikes per click (Fig. $11 A$ ), whereas spontaneously active afferents produced bursts of spikes (Fig. $11 B, C$ ). The influence of clicks on neurons with the highest spontaneous rates was a temporal reorganization of the spikes rather than an increase in spike rate.

When peristimulus histograms were examined for single click stimulation, we noted that the bursting afferents seemed to ring, producing peaks at regular intervals that corresponded roughly to the period of the BF (Fig. 11C). In contrast, many entrained afferents produced a more chaotic pattern of PSTH peaks (Fig. $11 B$ ). However, there was considerable variability in these single click responses, and click-response type did not map reliably with the clusters discussed above on the basis of tone responses.

All the afferents provided a faithful temporal representation of clicks trains and thus should also encode well the grunts of the courtship display. Neurons lacking spontaneous activity (silent) typically fired a single spike per click throughout the train (Fig. $12 \mathrm{Al}$ ), and consequently, the interspike intervals fell in a tight distribution around the train period (ICI) (Fig. 12A2). Additionally, the distribution generated by measuring all of the intervals, not just between adjacent spikes but between all peristimulus spikes (all-order interspike intervals), revealed intervals that were multiples of the click train period (Fig. 12A3). This all-order interval analysis was particularly useful for examining the temporal structure of the spike trains produced by spontaneously active afferents.

The entrainment of spontaneously active afferents during click trains was not as apparent in raster and PST plots (Fig. 12B1) as it was for the silent neurons. Nevertheless, the temporal structure of the spike train was clearly modulated, shifting the ISI distribution from a unimodal spontaneous distribution to a bimodal driven distribution (Fig. 12B2). The emergence of intervals corresponding to the ICI of the stimulus was revealed by the all-order ISI histogram where there were distinct peaks separated by the ICI of the click train (Fig. 12B3).

Afferents encoded a broad range of click train periods and were never selective for particular ICIs (range $=10-80 \mathrm{msec}$ ). Because the number of clicks per constant duration train increased with ICI, the function relating spike rate to ICI for afferents without spontaneous activity showed a monotonic decrease with increasing ICI for the train (Fig. 12A4, left). The function relating spikes per click to the ICI was flat (Fig. 12A4, right). For spontaneously active neurons, the evoked spike rate remained fairly constant for all ICIs, whereas the spikes per click increased as the ICI increased (Fig. 12B4). In contrast, about one-third of midbrain neurons exhibit interval selectivity, showing a highly facilitated response for a narrow range of interclick intervals (Crawford, 1997b). 
PSTH
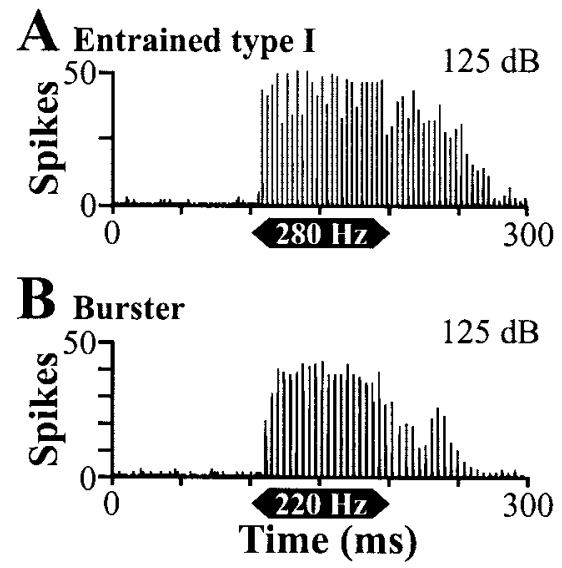

Period Histogram
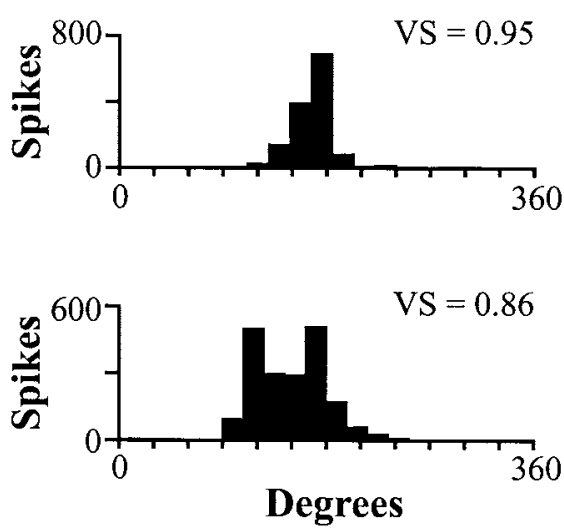

ISIH
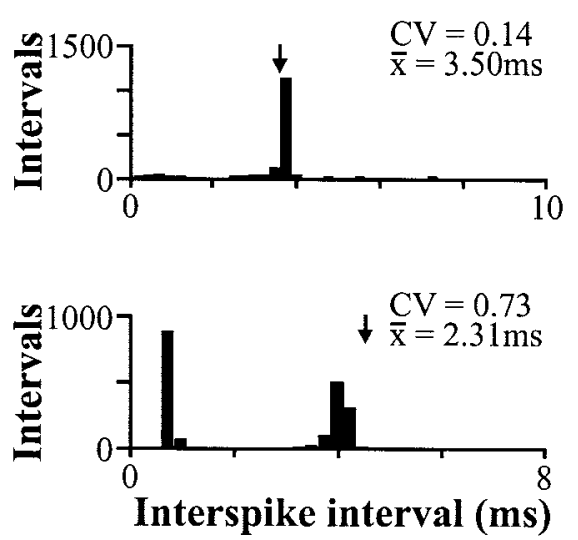

Figure 9. Temporal representation of tone bursts. PSTHs, period histograms, and ISIHs were constructed from spikes times that occurred during 50 presentations of the same tone burst at $125 \mathrm{~dB}$. A, Entrained type I; $B$, bursting. Arrows in the ISIH point to the period of tone. PSTH, $100 \mu$ sec bins; period histograms, $18^{\circ}$ bins; ISIH, $500 \mu \mathrm{sec}$ bins.

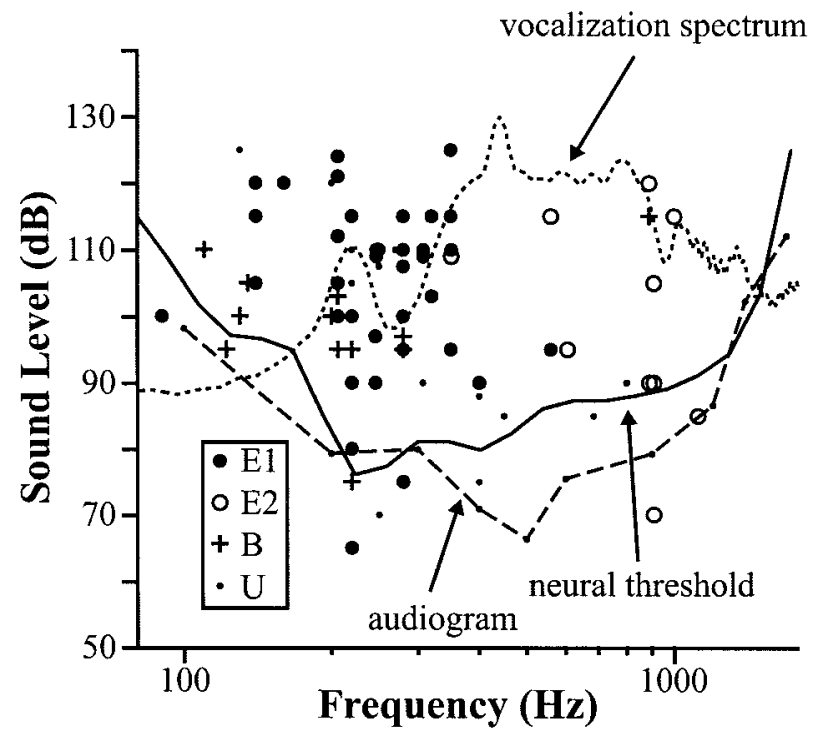

Figure 10. Distribution of characteristic frequencies and their corresponding best sensitivities. Each CF and BS was measured from an FTC (Fig. 8A1,B1, arrows). The dashed line is the behavioral audiogram (Marvit and Crawford, 2000b), and the dotted line is the frequency spectrum of the male vocalization (Crawford et al., 1997). The solid line connects the neural thresholds estimated from the 90th percentile of all the tuning curve values at each frequency. Note that the neural thresholds were not only based on the thresholds at CF (i.e., the BS data points) but were calculated from the values of all FTCs that traversed a given frequency. At some frequencies there were FTCs of certain neurons that were more sensitive than any neuron with a $\mathrm{CF}$ at that particular frequency.

\section{DISCUSSION}

This analysis of primary afferents has provided a view of the initial neural representation of sounds generated by a simple vertebrate ear and delivered to central computational circuits. The morphology of the ear, and the physiology of the midbrain, had led us to suspect that the feature selective responses of the midbrain were computed from an initial temporal representation created at the ear (Crawford, 1997b). The present data show that afferents provide an excellent temporal representation of acoustic stimuli but are not feature selective. The characteristic frequencies of these neurons were distributed within the measured audiogram for the animal, and tuning curves were generally quite broad.

Previous studies of the primary auditory afferents of other fishes, primarily goldfish, have focused on characteristics of the FTC, the position of the CF in particular, as a basis for defining physiological types of afferents (Furukawa and Ishii, 1967; Fay and Ream, 1986). However, we included temporal properties of spike trains in our classification of afferents because they are probably particularly important for central processing in the fish auditory system (Fay, 1978, 1982; Crawford, 1997b; McKibben and Bass, 1999; Bodnar et al., 2001).

Our entrained afferents (especially type II) were most like the goldfish high-frequency follower afferents (S1), and the bursters were most like low-frequency afferents (S2) of Furukawa and Ishii (1967). Fay and Ream (1986) identified four classes of goldfish afferents on the basis of the FTC, three of which were classified as tuned types. These tuned fibers had broad FTCs, with 95\% having $\mathrm{Q}_{10 \mathrm{~dB}} \leq 1.2$; the afferents of goldfish are thus more weakly tuned than those of mormyrids, but like mormyrids do show a preponderance of CFs near $200 \mathrm{~Hz}$ (Fay, 1978; Fay and Ream, 1986). On the basis of a comparison of CF distributions [BF in Fay and Ream (1986)], our bursting afferents were comparable to the low-frequency type in goldfish, the entrained type I afferents were comparable to the medium-frequency type in goldfish, and the entrained type II afferents were comparable to the highfrequency type in goldfish. The relationship between $\mathrm{CF}$ and RLF slope that we observed here was also similar to that observed in goldfish by Fay and Ream (1986), with RLF slope increasing with higher CFs (Fig. 12). Because goldfish do not make communication sounds, their CFs cannot be easily related to the acoustic behavior.

In midshipman fish, the BFs of auditory afferents matched the relatively narrow vocalization spectrum for this species (McKibben and Bass, 1999). The distribution of BFs for the midshipman $(60-300 \mathrm{~Hz})$ was narrower than that of mormyrids or goldfish. Midshipman afferents entrained well to low-frequency tones $(<200 \mathrm{~Hz})$ and amplitude modulated signals (amplitude modulation rate $<10 \mathrm{~Hz}$ ) (McKibben and Bass, 2001). 

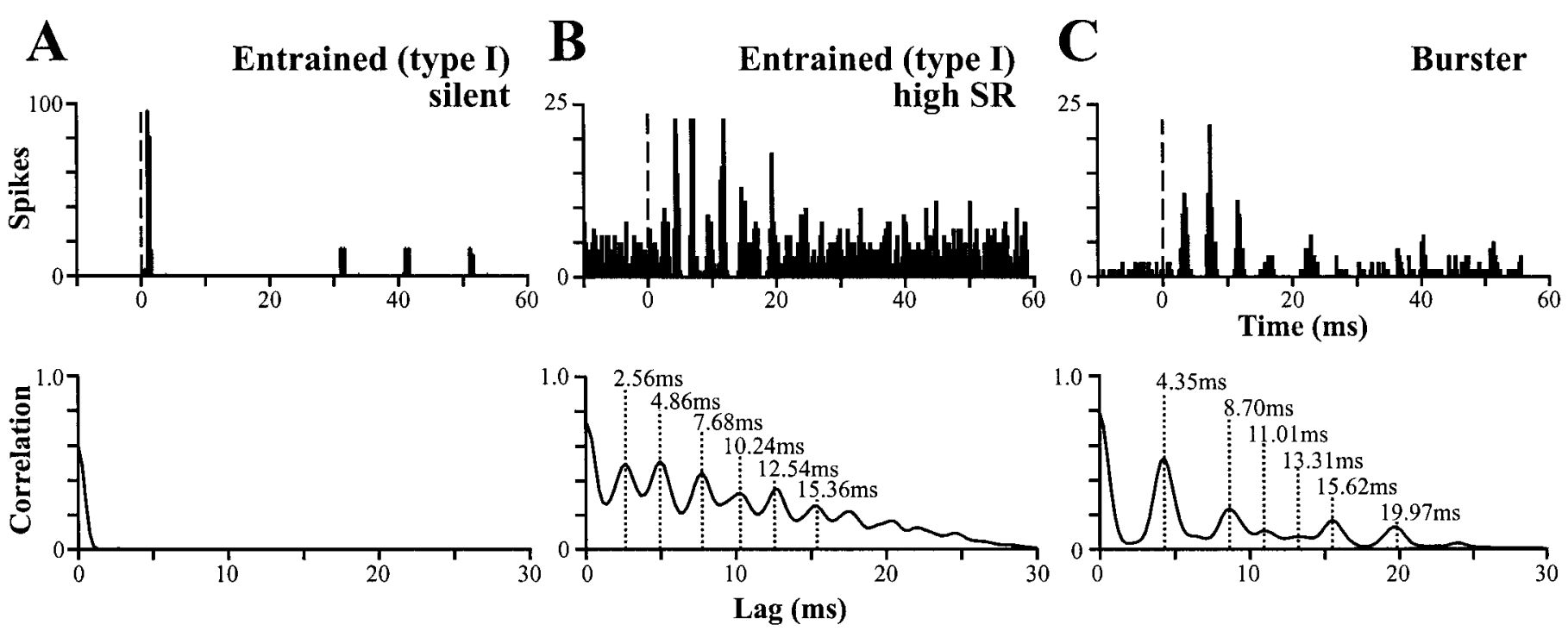

Figure 11. Examples of responses to single clicks. $A$, PSTH for a silent entrained type I neuron and its corresponding autocorrelation histogram (192 trials). $B$, PSTH for a spontaneously active entrained type I neuron and its corresponding autocorrelation histogram ( 32 trials). $C$, PSTH for a bursting neuron and its corresponding autocorrelation histogram (64 trials). The dashed lines represent the onset of the click stimulus. Note the orderly discharge pattern in the PSTH for the burster $(C)$ compared with the chaotic response of the spontaneously active entrained neuron $(B)$. The peaks in the autocorrelation function for the bursting neuron $(C$, right $)$ occurred at regularly spaced intervals, whereas those for the entrained neuron $(B$, right $)$ were not regularly spaced. All clicks were presented at a suprathreshold level of $125 \mathrm{~dB}$. PSTH bins, $500 \mu \mathrm{sec}$.

\section{Entrained afferents}

Entrainment of afferent spikes in mormyrids appears to be exceptional in two ways. First, the degree of synchrony is very high, usually $>0.9$, whereas synchrony in other vertebrate auditory systems is usually less (Palmer and Russell, 1986; Hill et al., 1989; Joris et al., 1994; Koppl, 1997). Second, many of these afferents entrain essentially perfectly up to $1.0 \mathrm{kHz}$, whereas mammalian and avian afferents skip cycles, failing to entrain when stimulus frequencies exceed $300 \mathrm{~Hz}$ (Kiang, 1965; Joris et al., 1994). Examples of sustained firing rates of $>1000$ spikes per second (Fig. 2) are uncommon and comparable to those reported for intralaminar thalamocortical cells (1000 spikes per second) (Steriade et al., 1993) and spinal Renshaw cells $(1500 \mathrm{~Hz})$ (Walmsley and Tracey, 1981).

The entrained afferents of mormyrids could represent a specialization for temporal computation. The interval distributions are unimodal, thus lacking the ambiguities of other neurons that have excellent synchrony (high VS) but skip cycles (high CV). If faithfully relayed to the midbrain, this temporal code could be used in the computations producing interval selectivity for click trains (Crawford, 1997b) or in the generation of frequencyselective responses for tonal signals (Licklider, 1951; Simmons et al., 1996).

The ability to entrain at high frequencies $(>300 \mathrm{~Hz})$ could reflect specializations at the synapses formed between HCs and afferents. For example, the neurotransmitter pool might be relatively large, thus increasing EPSP size. This could increase the probability that a spike would be generated on each stimulus cycle (Trussell, 1997). Furthermore, a larger pool would reduce the likelihood of neurotransmitter depletion and thus allow the afferent to follow stimuli at higher frequencies, without skipping cycles. Convergence of multiple HCs on a single afferent could also increase the probability that at least one synapse would contribute to spike initiation on every cycle (Furukawa and Ishii, 1967). The primary afferents of fish are branched within the saccular epithelium, providing a morphological basis for conver- gent input from multiple HCs (Sento and Furukawa, 1987; Kozloski and Crawford, 1998; Edds-Walton et al., 1999; Edds-Walton and Popper, 2000). The bushy cells in the mammalian anterior ventral cochlear nucleus also entrain very precisely, and this have been hypothesized to arise from the convergence of multiple afferents on these cells (Rothman et al., 1993; Joris et al., 1994).

\section{Bursting afferents}

Because of their relatively shallow, nonsaturating, rate-level functions and wide dynamic ranges, the bursting afferents seem to be better suited for encoding intensity information than the other afferent types. Bursting afferents have bandwidths $\left(\mathrm{Q}_{10 \mathrm{~dB}}=1.57\right.$; quartile range $25-75 \%=1.23-2.34)$ that are about the same as those of the low CF (i.e., 100-1000 Hz) afferent fibers of other vertebrates [mammals (Kiang, 1965) and turtles (Crawford and Fettiplace, 1980)].

We suspect that the bursting physiology of these afferents, in response to tones near $200 \mathrm{~Hz}$, reflects intrinsic properties of the HCs as in turtles (Art et al., 1986; Eatock et al., 1993; Fettiplace and Fuchs, 1999). Tuning in turtle auditory afferents is a direct result of the electrically tuned HCs onto which the afferents form synapses (Crawford and Fettiplace, 1980). The frequency sensitivity of the isolated turtle HCs (Fettiplace and Crawford, 1978, 1980; Fettiplace and Fuchs, 1999) is similar to that of the tuned mormyrid afferents. The differences between mormyrid afferents (i.e., bursting vs entrained) may correspond to differences in the characteristics of the HCs that they innervate (Popper et al., 1993; Lanford et al., 2000). The different patterns of single click responses, including ringing, chaotic bursts, and single spikes, are consistent with the idea that there may be different $\mathrm{HC}$ types associated with the different afferent clusters.

The observation that there are both highly entrained neurons with steep RLFs and bursting neurons with shallow, nonsaturating RLFs suggests that there may be a segregation of time and intensity information that begins in the auditory nerve of these fish. Similar parallel processing of time and intensity has been 

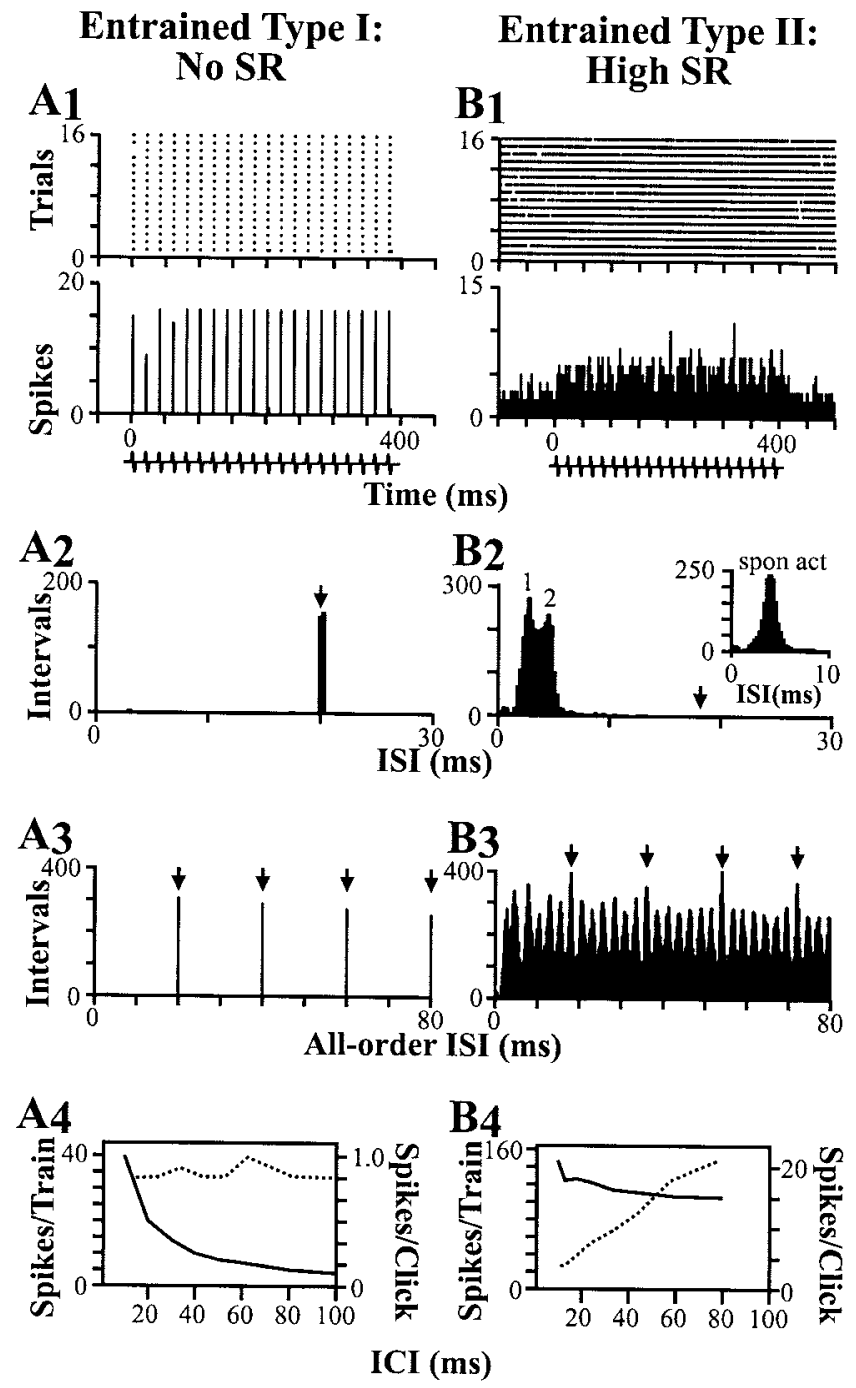

Figure 12. Responses to click trains. Raster plots and PSTH to click trains were constructed for silent $(A 1)$ and spontaneously active $(B 1)$ $(\mathrm{SR}=251$ spikes per second) entrained type I neurons. The interclick intervals $(I C I)$ of the click trains in $A 1$ and $B 1$ were 20 and $18 \mathrm{msec}$, respectively. ISIHs $(A 2, B 2)$ and all-order ISIHs $(A 3, B 3$; see Materials and Methods) were generated from the spike times in $A 1$ and $B 1$. The ISIH of the spontaneous activity is shown for comparison with the driven response (B2, inset). Arrows in the histograms point to the ICI. Histograms bins were $250 \mu \mathrm{sec}$. Mean spikes per click train (left axis, solid line) and per click (right axis, dotted line) were plotted as a function of ICI for silent $(A 4)$ and spontaneously active $(B 4)$ entrained type I neurons.

suggested in other auditory systems but is thought to begin within the brainstem nuclei of birds and mammals (Koppl et al., 2000). However, differentiation of physiological response types in the auditory nerve, suited to processing distinct components of communication sounds, has been suggested previously in frogs $(\mathrm{Ca}-$ pranica and Moffat, 1975; Narins and Capranica, 1980; Rose and Brenowitz, 1997).

\section{Transformations of primary afferent encoding}

The initial afferent representation of sound is markedly transformed as revealed by comparisons with the physiology of the second order medullary nucleus (Kozloski and Crawford, 2000) and auditory midbrain (Crawford, 1993, 1997b; Kozloski and Crawford, 1998). One of the most striking transformations is the emergence of selectivity for interclick intervals in the midbrain.
Afferents produced single spikes, or bursts, that were synchronized to each click, and none of the afferents was interval selective. In contrast, approximately one-third of midbrain neurons exhibit interval selectivity, showing a highly facilitated response for a narrow range of interclick intervals (Crawford, 1997b).

A second transformation was revealed by the emergence of tone frequency selectivity in the midbrain. The response areas of primary afferents were typically very broad. They produced one spike on each cycle of a tone, over a range of periods that extended from $\sim 10 \mathrm{msec}$ to just $<1.0 \mathrm{msec}$ ( $100 \mathrm{~Hz}$ to just $>1.0$ $\mathrm{kHz}$ ). This type of response area was not observed in the midbrain. In contrast, midbrain neurons often had narrow-band excitatory response areas and flanking regions of inhibition [see also Lu and Fay (1993)]. The tuning curves were spindle shaped, and neurons were relatively insensitive to tone intensity. These level tolerant neurons resemble some of the neurons in the midbrain of cats, frogs, and bats (Katsuki et al., 1958; Fuzessery and Feng, 1982; Suga, 1995).

All of the responses in the auditory nerve were sustained for the duration of a tone stimulus, but midbrain neurons have either onset or phasic responses, and some have delayed inhibition followed by off responses. Midbrain neurons also show weaker entrainment, attributable to both poor synchrony and cycle skipping. Thus, the precise temporal representation of sound is diminished in the midbrain. Afferent spike rates always increased as a monotonic function of stimulus intensity, whereas nonmonotonic rate-level functions were common among midbrain neurons.

\section{REFERENCES}

Art JJ, Crawford AC, Fettiplace R (1986) Electrical resonance and membrane currents in turtle cochlear hair cells. Hearing Res 22:31-36.

Batschelet E (1981) Circular statistics in biology. New York: Academic.

Bodnar DA, Bass AH (1997) Temporal coding of concurrent acoustic signals in auditory midbrain. J Neurosci 17:7553-7564.

Bodnar DA, Bass AH (2001) Coding of concurrent vocal signals by the auditory midbrain: effects of duration. J Comp Physiol [A] 187:381-391.

Bratton BO, Kramer B (1989) Patterns of the electric discharge during courtship and spawning in the mormyrid fish, Pollimyrius isidori. Behav Ecol Sociobiol 24:349-368.

Capranica RR, Moffat AJM (1975) Selectivity of the peripheral auditory system of spadefoot toads Scaphiopus couchi for sounds of biological significance. J Comp Physiol 100:231-249.

Crawford AC, Fettiplace R (1980) The frequency selectivity of auditory nerve fibres and hair cells in the cochlea of the turtle. J Physiol 306:79-125.

Crawford JD (1993) Central auditory neurophysiology of a soundproducing mormyrid fish: the mesencephalon of Pollimyrus isidori. J Comp Physiol 172:1-14.

Crawford JD (1997a) Feature-detecting auditory neurons in the brain of a sound-producing fish. J Comp Physiol [A] 180:439-450.

Crawford JD (1997b) Hearing and acoustic communication in the mormyrid electric fishes. Mar Fresh Behav Physiol 29:1-21.

Crawford JD, Hagedorn MM, Hopkins CD (1986) Acoustic communication in an electric fish, Pollimyrus isidori (Mormyridae). J Comp Physiol [A] 159:297-310.

Crawford JD, Cook AP, Heberlein AS (1997a) Bioacoustic behavior of African fishes (Mormyridae): potential cues for species and individual recognition in Pollimyrus. J Acoust Soc Am 102:1200-1212.

Crawford JD, Jacob P, Bénech V (1997b) Sound production and reproductive ecology of strongly acoustic fish in Africa: Pollimyrus isidori, Mormyridae. Behaviour 134:677-725.

Eatock RA, Saeki M, Hutzler MJ (1993) Electrical resonance of isolated hair cells does not account for acoustic tuning in the free-standing region of the alligator lizard's cochlea. J Neurosci 13:1767-1783.

Edds-Walton PL, Popper AN (2000) Dendritic arbors on the saccule and lagena in the ear of the goldfish, Carassius auratus. Hearing Res 141:229-242.

Edds-Walton PL, Fay RR, Highstein SM (1999) Dendritic arbors and central projections of physiologically characterized auditory fibers from the saccule of the toadfish, Opsanus tau. J Comp Neurol 411:212-238.

Fay RR (1974) Masking of tones by noise for the goldfish (Carassius auratus). J Comp Physiol Psychol 87:708-716.

Fay RR (1978) Coding of information in single auditory-nerve fibers of the goldfish. J Acoust Soc Am 63:136-146. 
Fay RR (1982) Neural mechanisms of an auditory temporal discrimination by the goldfish. J Comp Physiol [A] 147:201-216.

Fay RR, Passow B (1982) Temporal discrimination in the goldfish. J Acoust Soc Am 72:753-760.

Fay RR, Ream TJ (1986) Acoustic response and tuning in saccular nerve fibers of the goldfish (Carassius auratus). J Acoust Soc Am 79:18831895.

Fettiplace R, Crawford AC (1978) The coding of sound pressure and frequency in cochlear hair cells of the terrapin. Proc R Soc Lond B Biol Sci 203:209-218.

Fettiplace R, Crawford AC (1980) The origin of tuning in turtle cochlear hair cells. Hearing Res 2:447-454.

Fettiplace R, Fuchs PA (1999) Mechanisms of hair cell tuning. Annu Rev Physiol 61:809-834.

Fletcher LB, Crawford JD (2001) Acoustic detection by soundproducing fishes (Mormyridae): the role of gas-filled tympanic bladders. J Exp Biol 204:175-183.

Furukawa T, Ishii Y (1967) Neuorophysiological studies on hearing in goldfish. J Neurophysiol 30:1377-1403.

Fuzessery ZM (1982) Frequency selectivity in the anuran auditory midbrain: single unit responses to single and multiple tone stimulation. J Comp Physiol [A] 146:471-484.

Goldberg JM, Brown PB (1969) Response of binaural neurons of dog superior olivary complex to dichotic tonal stimuli: some physiological mechanisms of sound localization. J Neurophysiol 32:613-636.

Hill KG, Mo J, Stange G (1989) Induced suppression of spike responses to tone-on-noise stimuli in the auditory nerve of the pigeon. Hearing Res 39:49-62.

Howell DC (1992) Statistical methods for psychology, Ed 3. Belmont, NY: Duxbury.

Joris PX, Carney LH, Smith PH, Yin TC (1994) Enhancement of neural synchronization in the anteroventral cochlear nucleus. I. Responses to tones at the characteristic frequency. J Neurophysiol 71:1022-1036.

Katsuki Y, Sumi T, Uchiyama H, Watanabe T (1958) Electic responses of auditory neurons in cat to sound stimulation. J Neurophysiol 21:569-588.

Kawasaki M, Guo YX (1996) ) Neuronal circuitry for comparison of timing in the electrosensory lateral line lobe of the African wave-type electric fish Gymnarchus niloticus. J Neurosci 16:380-391.

Kiang NY-S (1965) Discharge patterns of single fibers in the cat's auditory nerve, Ed 1. Cambridge, MA: MIT.

Koppl C (1997) Phase locking to high frequencies in the auditory nerve and cochlear nucleus magnocellularis of the barn owl, Tyto alba. J Neurosci 17:3312-3321.

Koppl C, Manley GA, Konishi M (2000) Auditory processing in birds. Curr Opin Neurobiol 10:474-481.

Kozloski J, Crawford JD (1998) Functional neuroanatomy of auditory pathways in the sound-producing fish Pollimyrus. J Comp Neurol 401:227-252.

Kozloski J, Crawford JD (2000) Transformations of an auditory temporal code in the medulla of a sound-producing fish. J Neurosci 20:2400-2408.

Lanford PJ, Platt C, Popper AN (2000) Structure and function in the saccule of the goldfish (Carassius auratus): a model of diversity in the non-amniote ear. Hearing Res 143:1-13.

Licklider JCR (1951) A duplex theory of pitch perception. Experientia 7:128-134.

Lu Z, Fay RR (1993) Acoustic response properties of single units in the torus semicircularis of the goldfish, Carassius auratus. J Comp Physiol [A] 173:33-48.
Marvit P, Crawford JD (2000a) Auditory discrimination in a soundproducing electric fish (Pollimyrus): tone frequency and click-rate difference detection. J Acoust Soc Am 108:1819-1825.

Marvit P, Crawford JD (2000b) Auditory thresholds in a soundproducing electric fish (Pollimyrus): behavioral measurements of sensitivity to tones and click trains. J Acoust Soc Am 107:2209-2214.

McCormick CA, Popper AN (1984) Auditory sensitivity and psychophysical tuning curves in the elephant nose fish. J Comp Physiol 155:753-761.

McKibben JR, Bass AH (1999) Peripheral encoding of behaviorally relevant acoustic signals in a vocal fish: single tones. J Comp Physiol [A] 184:563-576.

McKibben JR, Bass AH (2001) Peripheral encoding of behaviorally relevant acoustic signals in a vocal fish: harmonic and beat stimuli. J Comp Physiol [A] 187:271-285.

Moller AR (1970) The use of correlational analysis in processing neuroelectric data. In: Progress in brain research, computers and brains (Schade JP, Smith J, eds), pp 87-99. Amsterdam: Elsevier.

Narins PM, Capranica RR (1980) Neural adaptations for processing the two-note call of the Puerto Rican tree frog, Eleutherodactylus coqui. Brain Behav Evol 17:48-66.

Palmer AR, Russell IJ (1986) Phase-locking in the cochlear nerve of the guinea-pig and its relation to the receptor potential of inner hair-cells. Hearing Res 24:1-15.

Perkel DH, Gerstein GL, Moore GP (1967) Neuronal spike trains and stochastic point processes. II. Simultaneous spike trains. Biophys J 7:419-440.

Popper AN, Saidel WM, Chang JS (1993) Two types of sensory hair cell in the saccule of a teleost fish. Hearing Res 64:211-216.

Rigley L, Marshall J (1973) Sound production by the elephant nose fish, Gnathonemus petersii (Pices, Mormyridae). Copeia 1973:134-135.

Rose GJ, Brenowitz EA (1997) Plasticity of aggressive thresholds in Hyla regilla: discrete accommodation to encounter calls. Anim Behav 53:353-361.

Rothman JS, Young ED, Manis PB (1993) Convergence of auditory nerve fibers onto bushy cells in the ventral cochlear nucleus: implications of a computational model. J Neurophysiol 70:2562-2583.

Sento S, Furukawa T (1987) Intra-axonal labeling of saccular afferents in the goldfish, Carassius auratus: correlations between morphological and physiological characteristics. J Comp Neurol 258:352-367.

Simmons AM, Shen Y, Sanderson MI (1996) Neural compuational basis for periodicity extraction in frog peripheral auditory system. Audit Neurosci 2:109-133.

Steriade M, Dossi RC, Contreas D (1993) Electrophysiological properties of intralaminar thalamocortical cells discharging rhythmic $(\sim 40$ $\mathrm{Hz}$ ) spike-bursts at $\sim 1000 \mathrm{~Hz}$ during waking and rapid eye movement sleep. Neuroscience 56:1-9.

Stipetic E (1939) Über das Gehörorgan der Mormyriden. Z Vgl Physiologie 26:740-752.

Suga N (1995) Sharpening of frequency tuning by inhibition in the central auditory system: tribute to Yusuji Katsuki. Neurosci Res 21:287299.

Trussell LO (1997) Cellular mechanisms for preservation of timing in central auditory pathways. Curr Opin Neurobiol 7:487-492.

Walmsley B, Tracey DJ (1981) An intracellular study of Renshaw cells. Brain Res 223:170-175.

Young ED, Robert JM, Shofner WP (1988) Regularity and latency of units in ventral cochlear nucleus: implications for unit classification and generation of response properties. J Neurophysiol 60:1-29. 